Pacific Journal of Mathematics

LATTICES WHOSE CONGRUENCES FORM A BOOLEAN 


\section{LATTICES WHOSE CONGRUENCES FORM A BOOLEAN ALGEBRA}

\section{Peter Crawley}

1. Introduction. The structure of a lattice $L$, in particular its representation as direct or subdirect unions, depends heavily on the structure of $\Theta(L)$, its lattice of congruence relations. Thus a natural problem which arises is that of determining those lattices whose congruence lattices have certain specified properties. Two problems of this general type are considered here.

The first problem involves the sequence of iterated congruence lattices. If $D$ is a distributive lattice, then as is well known, $D$ is isomorphic with a sublattice of $\Theta(D)$. Hence the iterations of $\Theta(D)$ form an ascending chain, $D \leq \Theta(D) \leq \Theta(\Theta(D)) \leq \cdots$. We shall show that this chain terminates if and only if $D$ is finite, thus answering a question of Morgan Ward. This result is a consequence of the theorem stating that if $L$ is an arbitrary lattice, then $L \cong \theta(L)$ if and only if $L$ is a finite boolean algebra. The proof of the latter statement rests on the following embedding theorem: if $D$ is a distributive lattice of infinite cardinality $\alpha$, then every distributive lattice of cardinality at most $2_{\infty}$ can be embedded in $\theta(\Theta(\Theta(\Theta(D))))$.

The second problem considered is that of characterizing those lattices $L$ for which $\Theta(L)$ is a boolean algebra. ${ }^{1}$ This problem has been solved previously by Tanaka [5] and by Grätzer and Schmidt [3], however, neither of these solutions is given entirely in terms of the intrinsic structure of the lattice $L$. Here such an "intrinsic" characterization is obtained, which has the form of a finiteness condition on the lattice. Several applications of this result are given, which illustrate the finiteness nature of the condition of complementation of $\Theta(L)$.

The main tool used in this investigation is the compactly generated property of $\Theta(L)$ (see $\S 2$ ). This property is a generalization of the ascending chain condition, and many important consequences of the chain condition also hold in lattices which are compactly generated.

2. Preliminaries. Throughout this note the familiar notation and terminology is used. The unit and null elements of a lattice $L$ will be denoted by $u$ and $z$ respectively. A quotient $a / b$ is defined by $a / b=$ $\{x \in L \mid b \leq x \leq a\}$. We say $a / b$ is prime if $a$ covers $b$. Each of the quotients $a / a \cap b$ and $a \cup b / b$ is said to be a transpose of the other. And a quotient $c / d$ is weakly projective into $a / b$ if there exists a finite

Received July 11, 1959.

1 This is listed as Problem 72 in [1]. 
sequence of quotients $c / d=x_{0} / y_{0}, x_{1} / y_{1}, \cdots, x_{k} / y_{k}=a / b$ such that each $x_{i-1} / y_{i-1}$ is contained in a transpose of $x_{i} / y_{i}$. The quotient $c / d$ is projective to $a / b$ if a sequence $c / d=x_{0} / y_{0}, \cdots, x_{k} / y_{k}=a / b$ exists such that each $x_{i-1} / y_{i-1}$ is a transpose of $x_{i} / y_{i}$.

A lattice $L$ is atomic if for every element $x \in L, x>z$, there exists an atom $p \leq x . \quad L$ is said to be weakly atomic if every proper quotient of $L$ contains a prime quotient.

The following two lemmas, which we state without proof, will be useful in the work below. The first is well known.

Lemma 2.1. Let $a / b$ and $c / d$ be two quotients of a lattice $L$.

(a) If $L$ is modular and c/d is weakly projective into $a / b$, then there is a quotient $a^{\prime} / b^{\prime}$ contained in $a / b$ such that $c / d$ and $a^{\prime} / b^{\prime}$ are projective.

(b) If $L$ is distributive and $a / b$ and c/d are projective, then there exists a quotient e/f which is a transpose of both $a / b$ and $c / d$.

The second lemma is due to R. P. Dilworth [2, p. 349]. Let us say that a congruence $\theta$ collapses a quotient $a / b$ if $a \equiv b(\theta)$. Suppose $Q$ is a set of quotients of a lattice $L$. Set $a \equiv b\left(\theta_{Q}\right)$ if and only if there exists a finite sequence $a \cup b=x_{0} \geq x_{1} \geq \cdots \geq x_{k}=a \cap b$ such that each $x_{i-1} / x_{i}$ is weakly projective into some quotient of $Q$.

LEMmA $2.2 \quad \theta_{Q}$ is the congruence relation generated on the lattice $L$ by collapsing the quotients of $Q$.

For single quotients $a / b$, the congruence relation generated by collapsing $a / b$ will be denoted by $\theta_{a / b}$.

A fundamental property of $\Theta(L)$, the lattice of all congruences of a lattice $L$, is that of being compactly generated. An element $c$ in a complete lattice $L$ is said to be compact if for every subset $S \subseteq L$ such that $c \leq \bigcup S$ there exists a finite subset $S^{\prime} \subseteq S$ such that $c \leq \bigcup S^{\prime}$. A lattice $L$ is compactly generated if $L$ is complete and every element of $L$ is a join of compact elements. Since for any lattice $L$ the congruences $\theta_{a / b}$ are compact and generate $\Theta(L)$, we have the following important lemma.

Lemma 2.3. For every lattice $L, \Theta(L)$ is compactly generated.

LEMMA 2.4. Every compactly generated lattice is weakly atomic.

Proof. Suppose $a / b$ is a quotient of a compactly generated lattice $L$, and $c$ is a compact element of $L$ such that $c \leq a$. Then if $S \subseteq a / b$ and

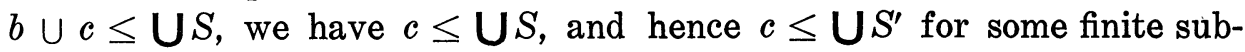
set $S^{\prime} \subseteq S$. But since $S^{\prime} \subseteq a / b, b \cup c \leq \cup S^{\prime}$, and hence $b \cup c$ is compact 
in $a / b$. Thus, since $x=\bigcup\{b \cup c \mid c$ compact, $c \leq x\}$ for each $x \in a / b$, the quotient $a / b$ is compactly generated.

Now suppose $a / b$ is a proper quotient of $L$. Let $c>b$ be a compact element of $a / b$, and let $P=\{x \in a / b \mid c>x \geq b\} . \quad P$ is non-empty since $b \in P$. Considering $P$ as a subpartially ordered set of $a / b$, let $M$ be a maximal chain of $P$, and let $q=\bigcup M$. Clearly $c \geq q$. Suppose $c=q$. Then since $c$ is compact, there exists a finite subset $\left\{x_{1}, \cdots, x_{k}\right\} \subseteq M$ such that $c \leq x_{1} \cup \cdots \cup x_{k}$. But $M$ is a chain and hence if $x_{j}$ is the largest of the elements $x_{1}, \cdots, x_{k}$, then $c \leq x_{j}$, contrary to $x_{j}<c$. Thus $c>q$. Suppose there is an element $y \in a / b$ such that $c>y>q$. Then the set-sum of $\{y\}$ and $M$ is a chain of $P$, contrary to the maximality of $M$. Hence $c$ covers $q$, and it follows that $L$ is weakly atomic.

Notice that from the preceding lemma it follows that every compactly generated complemented modular lattice $L$ is atomic. For suppose $x \in L, x>z$. Then there is a compact element $c$ such that $x \geq c$ and $c>z$. As shown above, there exists an element $q$ such that $c$ covers $q$. Let $p$ be a relative complement of $q$ in $c / z$. Since $c=p \cup q$ covers $q$, by modularity $p$ covers $p \cap q=z$. Hence $p$ is an atom contained in $x$, and $L$ is atomic.

The following lemma will also be needed.

Lemma 2.5. Let $L$ be a compactly generated distributive lattice. If $X \subseteq L$ and $y \in L$ are such that $x \cap x^{\prime}=y$ for every pair of distinct elements $x, x^{\prime} \in X$, then $L$ has a sublattice isomorphic with the boolean algebra of all subsets of $X$. If $X$ is the set of atoms of $L$ and $u=\bigcup X$, then $L$ is isomorphic with the boolean algebra of all subsets of $X$.

Proof. We first show that $L$ is infinitely join distributive. Let $A$ be a subset of $L$, and $b \in L$. Since $\bigcup b \cap A \leq b \cap \cup A$, to show that

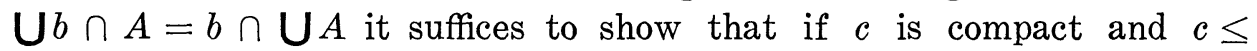
$b \cap \bigcup A$, then $c \leq \bigcup \mathbf{U} b A$. If $c$ is such an element, then $c \leq b$ and $c \leq \bigcup A$. Since $c$ is compact, $c \leq a_{1} \cup \cdots \cup a_{k}$ for a finite number of elements $a_{1}, \cdots, a_{k} \in A$. Thus $c \leq b \cap\left(a_{1} \cup \cdots \cup a_{k}\right)=\left(b \cap a_{1}\right) \cup \cdots \cup$

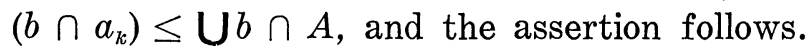

Suppose now that $U, V \subseteq X$. Let $S$ and $T$ be the set-sum and setproduct respectively of $U$ and $V$. Clearly $\cup U \cup \cup V=\cup S$. By infinite distributivity $(\bigcup U) \cap(\bigcup V)=\bigcup_{x \in U, x^{\prime} \in V} x \cap x^{\prime}=y \cup \bigcup T=\bigcup T$. (Here we take $\bigcup U=y$ if $U$ is empty.) Similarly it follows that $U U=$ $\bigcup V$ if and only if $U=V$. Hence $\{U U \mid U \subseteq X\}$ is a sublattice of $L$ isomorphic with the boolean algebra of all subsets of $X$. Suppose $X$ is the set of atoms of $L$, and $u=\bigcup X$. Then for each $a \in L, a=a \cap \bigcup X=$ $\bigcup a \cap X=\bigcup\{x \in X \mid x \leq a\}$. Thus $L=\{\bigcup U \mid U \subseteq X\}$, and hence $L$ is the boolean algebra of all subsets of $X$. 
3. Chain of congruence lattices. If $A$ is an ideal of a lattice $L$, then $A$ gives rise to the congruence $\theta_{A}$ generated by collapsing the quotients of $A$. It is well known and quite easily shown that if $D$ is $a$ distributive lattice, then the mapping $A \rightarrow \theta_{A}$ is an isomorphism of the lattice of ideals of $D$ into $\Theta(D)$. Moreover, coverings in the lattice of ideals of $D$ are preserved under this mapping. This isomorphism we shall call the natural mapping of the lattice of ideals of $D$ into $\Theta(D)$. In particular, $D$ is isomorphic with a sublattice of $\Theta(D)$. Thus we have the ascending chain of congruence lattices $D \leq \Theta(D) \leq \Theta(\Theta(D)) \leq \cdots$. For convenience, we will denote $\Theta(\Theta(D))$ by $\Theta^{2}(D), \Theta(\Theta(\Theta(D)))$ by $\Theta^{3}(D)$, etc.

LEMMA 3.1. If $L$ is a distributive lattice, then $\Theta(L)$ is atomic if and only if $L$ is weakly atomic.

Proof. Suppose $p / q$ is a prime quotient of $L$. If a non-trivial congruence $\theta_{a / b}$ is such that $\theta_{a / b} \leq \theta_{p / q}$, then by Lemma 2.2, $a / b$ contains a quotient $a / b^{\prime}$ such that $a / b^{\prime}$ is weakly projective into $p / q$. But $p / q$ is prime and hence by Lemma 2.1 (a), $a / b^{\prime}$ and $p / q$ are projective. Thus $\theta_{p / q}=\theta_{a / b^{\prime}} \leq \theta_{a / b} \leq \theta_{p / q}$, whence it follows that $\theta_{p / q}$ is an atom of $\Theta(L)$. If $L$ is weakly atomic, then every quotient $a / b$ contains a prime quotient $p / q$. Thus $\theta_{a / b}$ contains the atom $\theta_{p / q}$, and hence $\Theta(L)$ is atomic.

Now suppose $\theta(L)$ is atomic. If $\theta$ is an atom of $\Theta(L)$, then for some quotient $p / q$ in $L, \theta=\theta_{p / q}$. Suppose $p / q$ is not prime, that is, for some $r \in L, p>r>q$. Since $\theta_{p / q}$ is an atom, $\theta_{p / q}=\theta_{p / r}=\theta_{r / q}$, and hence by Lemmas 2.2 and 2.1 (a), $p / q$ contains a proper quotient $p / s$ and $r / q$ contains a proper quotient $g / h$ such that $p / s$ and $g / h$ are projective. By Lemma 2.1 (b), a quotient $e / f$ exists which is a transpose of both $p / s$ and $g / h$. Suppose $e \cup s=p, e \cap s=f$, and $f \cup g=e, f \cap g=h$. Then $s \geq f$, and hence $s \geq g \cup f=e$. Thus $p=s \cup e=s$, contrary to $p>s$. Similarly, the other conditions for $e / f$ to be a transpose of both $p / s$ and $g / h$ are impossible. Thus $p$ must cover $q$, and hence every atom of $\Theta(L)$ is generated by a prime quotient. Since $\Theta(L)$ is atomic, for every proper quotient $a / b, \theta_{a / b}$ contains an atom $\theta_{p / q}$ generated by the prime quotient $p / q$. Hence by Lemma 2.1 (a) and Lemma 2.2, $a / b$ contains a quotient $c / d$ which is projective to $p / q$. But in any modular lattice projective quotients are isomorphic, and thus $c / d$ is prime. Hence $L$ is weakly atomic.

Recall that a congruence $\theta$ on a lattice $L$ is completely irreducible if $\bigcap\{\phi \in \theta(L) \mid \phi>\theta\} \neq \theta$. The image of $L$ under any completely irreducible congruence is a subdirectly irreducible lattice.

LEMMA 3.2. Let $\theta$ be a non-trivial compact congruence on a lattice $L$. Then there exists a completely irreducible congruence $\theta^{*}$ on $L$ such that $\theta^{*} ¥ \theta$. 
Proof. Let $P$ be the partially ordered set of all congruences $\phi$ on

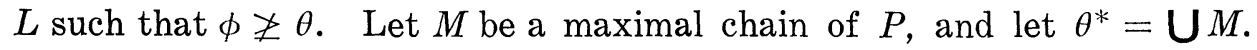
If $\theta \leq \theta^{*}$, then since $\theta$ is compact, $\theta \leq \phi_{1} \cup \cdots \cup \phi_{k}$ for a finite number of elements $\phi_{1}, \cdots, \phi_{k} \in M$. But $M$ is a chain, whence if $\phi_{j}$ is the largest element among $\phi_{1}, \cdots, \phi_{k}$, then $\theta \leq \phi_{j}$, a contradiction. Hence $\theta^{*} ¥ \theta$. Suppose $\bigcap\left\{\phi \in \Theta(L) \mid \phi>\theta^{*}\right\}=\theta^{*}$. Then for some $\phi_{0}>\theta^{*}$ it must be that $\phi_{0} \geq \theta$. But then the set-sum of $\left\{\phi_{0}\right\}$ and $M$ is a chain of $P$ properly containing $M$, contrary to the maximality of $M$. Hence $\theta^{*}$ is completely irreducible, and the lemma follows.

If $D$ is an infinite distributive lattice, then either $D$ has an infinite acsending chain or $D$ has an infinite descending chain. Suppose $D$ has an infinite ascending chain, $a_{0}<a_{1}<\cdots<a_{i}<\cdots$. Then as seen in the proof of Lemma 3.1, the congruences $\theta_{a_{i} / a_{i-1}}$ are disjoint, that is, if $i \neq j$ then $\theta_{a_{i} / a_{i-1}} \cap \theta_{a_{j} / a_{j-1}}$ is the null congruence on $D$. Hence by Lemma 2.5, $\Theta(D)$ contains a sublattice isomorphic with the complete atomic boolean algebra with a countable number of atoms. Since every countable distributive lattice can be embedded in this boolean algebra (see the proof of Theorem 3.1), every countable distributive lattice can be embedded in $\Theta(D)$. Thus if $D$ is an infinite distributive lattice, then for every countable distributive lattice $L, \Theta(L)$ contains a sublattice isomorphic with $L$. Suppose now that $D$ is the chain of real numbers. Then since every set of disjoint intervals of $D$ is at most countable, it follows that no distributive lattice with more than a countable number of atoms can be embedded in $\Theta(D)$. Thus if $D$ is a distributive lattice of infinite cardinality $\alpha$, then in general every distributive lattice of cardinality $\alpha$ cannot be embedded in $\Theta(D)$.

THeOREm 3.1. Let $D$ be a distributive lattice of infinite cardinality $\alpha$. If $L$ is any distributive lattice of cardinality at most $2^{\alpha}$, then $\Theta^{4}(D)$ contains a sublattice isomorphic with $L$.

Proof. Since $\Theta(D)$ is compactly generated, it is weakly atomic by Lemma 2.4. Hence by Lemma 3.1, $\Theta^{2}(D)$ is atomic. Let $M$ be the set of atoms of $\Theta^{2}(D)$, and let $\beta$ be the cardinality of $M$. For every atom $\pi \in M$, let $\pi^{*}$ be a completely irreducible congruence on $\Theta(D)$ such that $\pi^{*} \nsucceq \pi$. Since every atom of $\Theta^{2}(D)$ is necessarily compact, such a completely irreducible congruence exists by Lemma 3.2. Then since $\Theta^{2}(D)$ is atomic, $\bigcap_{\pi \in M} \pi^{*}$ must equal the null congruence on $\Theta(D)$. Now the image of any distributive lattice under a completely irreducible congruence is the two-element lattice. Hence $\Theta(D)$ has a representation as a subdirect union of $\beta$ copies of the two-element lattice. Thus $\Theta(D)$ has cardinality at most $2^{\beta}$, and since $\Theta(D)$ contains $D$ as a sublattice, we must have $2^{\beta} \geq \alpha$.

In $\Theta^{2}(D)$ let $\mu=\mathrm{U} M$. Then the principal ideal $(\mu)$ of $\Theta^{2}(D)$ is 
isomorphic with the boolean algebra of all subsets of $M$, by Lemma 2.5. Now every ideal of $(\mu)$ is also an ideal of $\Theta^{2}(D)$. Thus under the natural mapping of the lattice of ideals of $\Theta^{2}(D)$ into $\Theta^{3}(D)$, the lattice of ideals of $(\mu)$ is mapped into $\Theta^{3}(D)$ in such a way that coverings in the lattice of ideals of $(\mu)$ are preserved. Thus if $\lambda \in \Theta^{3}(D)$ is the image of $\mu$ under the natural mapping, and $J \subseteq \Theta^{3}(D)$ is the set of all images under this mapping of the maximal ideals of $(\mu)$, then $\lambda$ covers each element $\sigma \in J$. A result of Tarski [6, p. 62] states that a complete atomic boolean algebra with $\beta$ atoms has $2^{2^{\beta}}$ maximal ideals. Thus $J$ has cardinality $2^{2^{2}}$. Suppose $\sigma_{1}, \sigma_{2} \in J$ with $\sigma_{1} \neq \sigma_{2}$. Then $\lambda / \sigma_{1}$ and $\lambda / \sigma_{2}$ generates congruence $\theta_{\lambda / \sigma_{1}}$ and $\theta_{\lambda / \sigma_{2}}$ on $\Theta^{3}(D)$. Suppose $\theta_{\lambda / \sigma_{1}}=\theta_{\lambda / \sigma_{2}}$. Then since $\lambda / \sigma_{i}$ are prime, by Lemma 2.1 there exists a prime quotient $\rho / \tau$ which is a transpose of both $\lambda / \sigma_{1}$ and $\lambda / \sigma_{2}$. Two possibilities arise: $\lambda \cap \tau=\rho$, $\lambda \cup \tau=\sigma_{1}=\sigma_{2}$; and $\sigma_{1} \cup \rho=\sigma_{2} \cup \rho=\lambda, \sigma_{1} \cap \rho=\sigma_{2} \cap \rho=\tau$. The first is impossible since $\sigma_{1} \neq \sigma_{2}$. If the second holds, then $\sigma_{1}=\sigma_{1} \cup\left(\sigma_{2} \cap \rho\right)=$ $\left(\sigma_{1} \cup \sigma_{2}\right) \cap\left(\sigma_{1} \cup \rho\right)=\lambda$, contrary to $\sigma_{1}<\lambda$. Hence $\theta_{\lambda / \sigma_{1}} \neq \theta_{\lambda / \sigma_{2}}$, and each of the prime quotients $\lambda / \sigma, \sigma \in J$, generates a unique minimal congruence on $\Theta^{3}(D)$. Thus $\Theta^{4}(D)$ has at least $2^{2^{\beta}}$ atoms, and hence by Lemma 2.5, $\Theta^{4}(D)$ contains a sublattice isomorphic with the complete atomic boolean algebra with $2^{\alpha}$ atoms.

To complete the proof if suffices to show that every distributive lattice of cardinality $\gamma$ can be embedded in a complete atomic boolean algebra with $\gamma$ atoms. Suppose $L$ is a distributive lattice of infinite cardinality $\gamma$. For each quotient $a / b$ of $L$, let $\theta_{a / b}^{*}$ be a completely irreducible congruence on $L$ such that $\theta^{*} \geq \theta_{a / b}$. Then $\bigcap_{a>b} \theta_{a / b}^{*}$ must equal the null congruence on $L$. Thus, since there are $\gamma$ pairs of elements $a>b$ in $L$, the lattice $L$ has a representation as a subdirect union of $\gamma$ copies of the two-element lattice. And hence $L$ is a sublattice of the complete atomic boolean algebra with $\gamma$ atoms. This completes the proof of the theorem.

Theorem 4.1 raises the question, can every distributive lattice of infinite cardinality $\alpha$ (or perhaps even $2^{\alpha}$ ) be embedded in $\Theta^{2}(D)$ or $\Theta^{3}(D)$ if $D$ has cardinality $\alpha$ ? Using the same techniques as above, it can be shown that if $\alpha$ is a limit cardinal such that $2^{\beta}<\alpha$ whenever $\beta<\alpha$, and $D$ is a distributive lattice of cardinality $\alpha$, then every distributive lattice of cardinality $\alpha$ can be embedded in $\Theta^{2}(D)$. Other than this, the question is open. ${ }^{2}$

The chain of congruence lattices can be extended transfinitely in the natural way. For these chains it is easy to show that if $D$ is an infinite distributive lattice, then any distributive lattice can be embedded in some transfinite iteration of $\Theta(D)$.

2 The following can be shown: the proposition that every distributive lattice of cardinality $\alpha$ can be embedded in $\Theta^{2}(D)$ if $D$ has cardinality $\alpha$, is equivalent to the proposition that every boolean algebra of cardinality $\alpha$ has at least $\alpha$ prime ideals. 
THEOREM 3.2. Let $L$ be an arbitrary lattice. Then $L$ is isomorphic with $\Theta(L)$ if and only if $L$ is a finite boolean algebra.

Proof. Suppose $L$ is infinite, say of cardinality $\alpha$. Then if $L \cong$ $\Theta(L)$, it follows that $L \cong \Theta^{4}(L)$. But then $L$ must have cardinality at least $2^{\alpha}$ by Theorem 3.1 , a contradiction. Hence if $L \cong \Theta(L)$, then $L$ is finite. Now for any finite distributive lattice $L, \Theta(L)$ is a finite boolean algebra of length equal to that of $L$. Hence $L \cong \Theta(L)$ if and only if $L$ is a finite boolean algebra.

CoROllaRY. If $D$ is a distributive lattice, then the ascending chain $D \leq \Theta(D) \leq \Theta^{2}(D) \leq \cdots$ terminates if and only if $D$ is finite.

4. Congruences forming boolean algebras. In this section we give a necessary and sufficient condition on $L$ for $\Theta(L)$ to be a boolean algebra. Our characterization rests on the notion minimal quotient. A proper quotient $a / b$ of a lattice $L$ is said to be minimal if for each proper quotient $c / d$ weakly projective into $a / b$ there exists a finite chain $a=x_{0}>x_{1}>\cdots>x_{k}=b$ such that each $x_{i-1} / x_{i}$ is weakly projective into $c / d$.

Theorem 4.1. Let $L$ be an arbitrary lattice. Then $\Theta(L)$ is a boolean algebra if and only if for each proper quotient $a / b$ of $L$ there exists $a$ finite chain $a=c_{0}>c_{1}>\cdots>c_{k}=b$ such that each $c_{i-1} / c_{b}$ is minimal.

Proof. Let $L$ be any lattice, and suppose $\theta$ is an atom of $\theta(L)$. Then $\theta$ must be generated by collapsing some quotient of $L$. Let $a / b$ be any quotient which generates $\theta$. Suppose $c / d$ is a proper quotient weakly projective into $a / b$. Then $\theta_{c / a} \leq \theta$, and since $\theta$ is an atom, $\theta_{c / a}=\theta$. Thus $a \equiv b\left(\theta_{c / a}\right)$ and hence a chain $a=x_{0}>x_{1}>\cdots>x_{k}=b$ exists such that each $x_{i-1} / x_{i}$ is weakly projective into $c / d$. Hence $a / b$ is minimal. Similarly, it follows that any minimal quotient of $L$ generates an atom of $\Theta(L)$.

Suppose now that for every proper quotient $a / b$ of a lattice $L$ there is a finite chain $a=c_{0}>c_{1}>\cdots>c_{k}=b$ such that each $c_{i-1} / c_{i}$ is minimal. Then each $c_{c_{i-1} / c_{i}}$ is an atom of $\Theta(L)$, and by Lemma 2.2, $\theta_{a / b}=$ $\theta_{c_{0} / c_{1}} \cup \cdots \cup \theta_{c_{k-1} / c_{k}}$. Thus every element of $\Theta(L)$ is a join of atoms, and hence by Lemma 2.5, $\Theta(L)$ is a boolean algebra. Conversely, suppose $\Theta(L)$ is a boolean algebra. Then since $\Theta(L)$ is compactly generated, by the remark following Lemma 2.4, $\Theta(L)$ is also atomic, and hence every element of $\Theta(L)$ is a join of atoms. Let $a / b$ be a proper quotient of $L$. Then $\theta_{a / b}$ is a join of atoms $\theta_{x_{j} / y_{j}}$ generated by minimal quotients $x_{j} / y_{j}$. By Lemma 2.2, there then exists a chain $a=c_{0}>c_{1}>\cdots>c_{k}=b$ such that each $c_{i-1} / c_{i}$ is weakly projective into some $x_{i} / y_{i}$. Now each $\theta_{c_{i-1} / c_{i}}$ 
is a non-trivial congruence and $\theta_{c_{i-1} / c_{i}} \leq \theta_{x_{i} / y_{i}}$; since $\theta_{x_{i} / y_{i}}$ is an atom of $f(L)$, we must have $\theta_{c_{i-1} / c_{i}}=\theta_{x_{i} / y_{i}}$. Hence, since $\theta_{c_{i-1} / c_{i}}$ is an atom, each of the quotients $c_{i-1} / c_{i}$ is minimal, and the proof of Theorem 4.1 is complete.

Notice that implicit in the proof above is the following: if $L$ has a unit and null element and $\Theta(L)$ is a boolean algebra, then $\Theta(L)$ is finite.

THeOREM 4.2. Let $L$ be a weakly atomic modular lattice. Then $\Theta(L)$ is a boolean algebra if and only if every quotient of $L$ is finite dimensional.

Proof. Let $c / d$ be any prime quotient of $L$. If a quotient $e / f$ is weakly projective into $c / d$, then by Lemma 2.1 and the fact that $c / d$ is prime, it follows that $c / d$ and $e / f$ are projective. Thus $c / d$ is a minimal quotient. Hence if every quotient of $L$ is finite dimensional, the conditions of Theorem 4.1 are satisfied, and $\Theta(L)$ is a boolean algebra.

Let $c / d$ be a minimal quotient of a modular lattice, and let $e / f$ be a prime quotient contained in $c / d$. $e / f$ is trivially weakly projective into $c / d$, and hence there exists a finite chain $c=x_{0}>x_{1}>\cdots>x_{k}=d$ such that each $x_{i-1} / x_{i}$ is weakly projective into $e / f$. But $e / f$ is prime, whence by Lemma 3.1 each $x_{i-1} / x_{i}$ is projective to $e / f$. In any modular lattice projective quotients are isomorphic, and hence each of the quotients $x_{i-1} / x_{i}$ is prime. Thus if $c / d$ is a minimal quotient of a modular lattice and $c / d$ contains a prime quotient, then $c / d$ is finite dimensional.

Now if $L$ is a weakly atomic modular lattice and $\Theta(L)$ is a boolean algebra, then for every proper quotient $a / b$ of $L$ there is a finite chain $a=c_{0}>c_{1}>\cdots>c_{k}=b$ such that each $c_{i-1} / c_{i}$ is minimal. As each $c_{i-1} / c_{i}$ is thus finite dimensional the quotient $a / b$ is finite dimensional, and the proof is complete.

Since every compactly generated lattice is weakly atomic and has a unit and a null element, the following theorem is an immediate consequence of Theorem 4.2.

THEOREM 4.3. Let $L$ be a compactly generated modular lattice. Then $\Theta(L)$ is a boolean algebra if and only if $L$ is finite dimensional. ${ }^{3}$

These results illustrate the finiteness nature of the condition of complementation on $\Theta(L)$. In fact, if $L$ is distributive, then a result of Hashimoto [4] states that $\Theta(L)$ is a boolean algebra if and only if the quotients of $L$ are finite dimensional. This, of course, no longer holds for modular lattices unless some additional finiteness condition is imposed;

${ }^{3}$ Since for any lattice $L$, the lattice $I(L)$ of all ideals of $L$ is compactly generated, this theorem contains a result of D. Edmondson, Bull. Amer. Math. Soc., vol. 62 (1956), p. 349, which states that for modular lattices $L, \Theta(I(L))$ is a boolean algebra if and only if $L$ is finite dimensional. 
the continuous geometries are well known examples.

One of the principal results of Dilworth [2] is that if $L$ is a relatively complemented lattice satisfying the ascending chain condition, then $\Theta(L)$ is a boolean algebra. This can be derived almost directly from Theorem 4.1. It is very easily shown that the conclusion of Lemma 2.1 (a) also holds for relatively complemented lattices. ${ }^{4}$ Thus, as in the modular case, every prime quotient of a relatively complemented lattice is minimal. Let $L$ be a relatively complemented lattice with the ascending chain condition, and let $a / b$ be a proper quotient of $L$. By the inductive form of the chain condition, we may assume that if $x>b$, then there is a finite chain $a=c_{0}>c_{1}>\cdots>c_{k}=x$ such that each $c_{i-1} / c_{i}$ is minimal. Let $d$ be a maximal element in $a / b$, and let $x$ be a relative complement of $d$ in $a / b$. Then since $a / d$ is prime and hence minimal, $x / b$ must minimal. Thus the condition of Theorem 4.1 is satisfied, and Dilworth's result follows.

\section{REFERENCES}

1. G. Birkhoff, Lattice Theory, rev. ed., Amer. Math. Soc. Colloq. Publ., vol. 25, 1948.

2. R. P. Dilworth, The structure of relatively complemented lattices, Ann. of Math., 51 (1950), 348-359.

3. G. Grätzer and E. T. Schmidt, Ideals and congruence relations in lattices, Acta Math. Acad. Sci. Hungar., 9 (1958), 137-175.

4. J. Hashimoto, Ideal theory for lattices, Math. Japonicae, 2 (1952), 149-186.

5. T. Tanaka, Cononical subdirect factorizations of lattices, J. Sci. Hiroshima Univ., ser

A, 16 (1952), 239-246.

6. A. Tarski, Ideale in vollständigen Mengenkörpern I, Fund. Math., 32 (1939), 45-63.

California Institute of Technology

${ }^{4}$ Cf., [2, p. 355]. 



\section{PACIFIC JOURNAL OF MATHEMATICS}

\section{EDITORS}

\author{
David Gilbarg \\ Stanford University \\ Stanford, California \\ F. H. Brownell \\ University of Washington \\ Seattle 5 , Washington
}

\author{
A. L. Whiteman \\ University of Southern California \\ Los Angeles 7, California \\ L. J. PAIGE \\ University of California \\ Los Angeles 24, California
}

\section{ASSOCIATE EDITORS}

\author{
E. F. BECKENBACH \\ T. M. CHERRY \\ D. DERRY
}
E. HEWITT
A. HORN
L. NACHBIN
M. OHTSUKA
H. L. ROYDEN
M. M. SCHIFFER

E. SPANIER

E. G. STRAUS

F. WOLF

\section{SUPPORTING INSTITUTIONS}

\author{
UNIVERSITY OF BRITISH COLUMBIA \\ CALIFORNIA INSTITUTE OF TECHNOLOGY \\ UNIVERSITY OF CALIFORNIA \\ MONTANA STATE UNIVERSITY \\ UNIVERSITY OF NEVADA \\ NEW MEXICO STATE UNIVERSITY \\ OREGON STATE COLLEGE \\ UNIVERSITY OF OREGON \\ OSAKA UNIVERSITY \\ UNIVERSITY OF SOUTHERN CALIFORNIA
}

\author{
STANFORD UNIVERSITY \\ UNIVERSITY OF TOKYO \\ UNIVERSITY OF UTAH \\ WASHINGTON STATE COLLEGE \\ UNIVERSITY OF WASHINGTON

AMERICAN MATHEMATICAL SOCIETY
CALIFORNIA RESEARCH CORPORATION
HUGHES AIRCRAFT COMPANY
SPACE TECHNOLOGY LABORATORIES
NAVAL ORDNANCE TEST STATION

Mathematical papers intended for publication in the Pacific Journal of Mathematics should be typewritten (double spaced), and the author should keep a complete copy. Manuscripts may be sent to any one of the four editors. All other communications to the editors should be addressed to the managing editor, L. J. Paige at the University of California, Los Angeles 24, California. ..

50 reprints per author of each article are furnished free of charge; additional copies may be obtained at cost in multiples of 50 .

The Pacific Journal of Mathematics is published quarterly, in March, June, September, and December. The price per volume (4 numbers) is $\$ 12.00$; single issues, $\$ 3.50$. Back numbers are available. Special price to individual faculty members of supporting institutions and to individual members of the American Mathematical Society: $\$ 4.00$ per volume; single issues, $\$ 1.25$.

Subscriptions, orders for back numbers, and changes of address should be sent to Pacific Journal of Mathematics, 2120 Oxford Street, Berkeley 4, California.

Printed at Kokusai Bunken Insatsusha (International Academic Printing Co., Ltd.), No. $6_{4}$ 2-chome, Fujimi-cho, Chiyoda-ku, Tokyo, Japan.

PUBLISHED BY PACIFIC JOURNAL OF MATHEMATICS, A NON-PROFIT CORPORATION

The Supporting Institutions listed above contribute to the cost of publication of this Journăl, but they are not owners or publishers and have no responsibility for its content or policies. 


\section{Pacific Journal of Mathematics}

\section{Vol. 10, No. $3 \quad$ November, 1960}

Glen Earl Baxter, An analytic problem whose solution follows from a simple

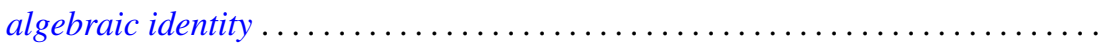

Leonard D. Berkovitz and Melvin Dresher, A multimove infinite game with linear payoff. .

Earl Robert Berkson, Sequel to a paper of A. E. Taylor ......................

Gerald Berman and Robert Jerome Silverman, Embedding of algebraic systems.... 767

Peter Crawley, Lattices whose congruences form a boolean algebra . . . . . ...... 777

Robert E. Edwards, Integral bases in inductive limit spaces . . . . . . . . . . . . . . .

Daniel T. Finkbeiner, II, Irreducible congruence relations on lattices . . . . . . . . . .

William James Firey, Isoperimetric ratios of Reuleaux polygons . . . . . . . . . . . 787

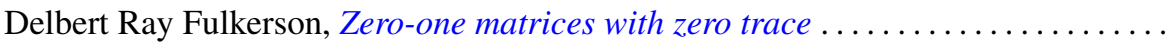

Leon W. Green, A sphere characterization related to Blaschke's conjecture........

Israel (Yitzchak) Nathan Herstein and Erwin Kleinfeld, Lie mappings in

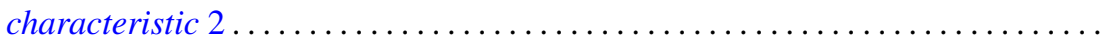

Charles Ray Hobby, A characteristic subgroup of a $p$-group .................

R. K. Juberg, On the Dirichlet problem for certain higher order parabolic

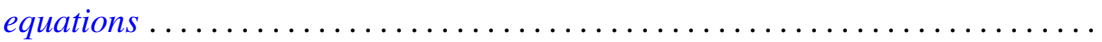

Melvin Katz, Infinitely repeatable games ......................

Emma Lehmer, On Jacobi functions . . . . . . . . . . . . . . . . . . . . . . . . .

D. H. Lehmer, Power character matrices

Henry B. Mann, A refinement of the fundamental theorem on the density of the sum of two sets of integers.

Marvin David Marcus and Roy Westwick, Linear maps on skew symmetric matrices: the invariance of elementary symmetric functions . .

Richard Dean Mayer and Richard Scott Pierce, Boolean algebras with ordered

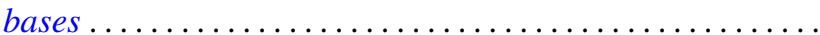

Trevor James McMinn, On the line segments of a convex surface in $E_{3} \ldots$

Frank Albert Raymond, The end point compactification of manifolds ..

Edgar Reich and S. E. Warschawski, On canonical conformal maps of regions of arbitrary connectivity

Marvin Rosenblum, The absolute continuity of Toeplitz's matrices...

Lee Albert Rubel, Maximal means and Tauberian theorems . .

Helmut Heinrich Schaefer, Some spectral properties of positive linear operators

Jeremiah Milton Stark, Minimum problems in the theory of pseudo-conformal transformations and their application to estimation of the curvature of the invariant metric.

Robert Steinberg, The simplicity of certain groups ...

Hisahiro Tamano, On paracompactness. .

Angus E. Taylor, Mittag-Leffler expansions and spectral theory .

Marion Franklin Tinsley, Permanents of cyclic matrices ...... . 